\title{
El impuesto óptimo al café: una evaluación de las diferentes alternativas*
}

\author{
Oscar E. Melhado
}

\section{INTRODUCCION}

Las recaudaciones fiscales provenientes del impuesto a la exportación del café son un componente principal de las recaudaciones totales en El Salvador. En los últimos diez anos han representado aproximadamente un $25 \%$ de las recaudaciones fiscales totales.

Sobra decir la importancia del rubro del café para la economía salvadorena. No sólo las finanzas públicas sino que toda la economla depende del sector cafetalero. A nivel de ingresos fiscales, no se puede prescindir de los ingresos generados por el gravamen a la exportación de café. En términos de equidad y redistribución, ese impuesto se presenta como una altemativa adecuada, dada las caracterlsticas del sector cafetalero.

Considerando las necesidades de recaudación del Estado y la incidencia redistributiva, se trata, por lo tanto, de gravar al café de la manera más eficiente; es decir, de modo tal que no implique un deterioro de la actividad cafetalera y no afecte adversamente el crecimiento económico. En este trabajo se analizan las distintas altemativas para

- Este articulo es un resumen de uno de los capitulos de la tesis doctoral del autor, titulada Taxation of Cofee Export and Behavior of Coffee Prices in El Salvador. La tesis fue presentada en Boston University en diciembre de 1990 . 
gravar al café y se ofrece una solución al problema del impuesto óptimo. Son tres las alternativas posibles para gravar al café que se consideran: el tradicional impuesto a la exportación, el impuesto al ingreso de los cafetaleros y un impuesto a la tierra. Nuestra contribución consiste en clarificar teóricamente la forma más eficiente para gravar al café.

Estudiamos el problema de una manera estática. En la primera sección el análisis se lleva a cabo asumiendo certidumbre en los precios. La segunda sección contiene una extensión de un análisis previo de la incertidumbre en la demanda de factores. La tercera sección se concentra en el problema del impuesto óptimo, dada la incertidumbre en los precios, considerando primero el uso de un solo tipo de impuesto y luego la combinación óptima de impuestos. Un supuesto importante para todo el análisis es la aversión al riesgo por parte de los cafetaleros; esto puede explicar algunos de los resultados obtenidos.

Este artículo puede ayudar a explicar los aspectos microeconómicos del impuesto al café en El Salvador. Sin embargo, es preciso advertir que el trabajo no está orientado hacia política económica, ya que dejamos afuera las restricciones administrativas enfrentadas por la distintas alternativas. Es importante senalar que la evasión y los problemas administrativos orientan el gravamen hacia el impuesto a la exportación. La implementación de un impuesto a la tierra o a las ganancias implica altos costos administrativos y motiva o hace más fácil la evasión. La omisión del problema de la evasión en nuestro modelo es razón suficiente para no considerar nuestras conclusiones orientadas hacia política económica. Nuestras conclusiones son, más bien, de naturaleza fundamentalmente teórica.

\section{LAS ALTERNATIVAS DE LA BASE DEL IMPUESTO AL CAFE ASUMIENDO CERTIDUMBRE EN EL PRECIO}

Diversos articulos han enfocado el problema de la equivalencia en modelos estáticos entre un impuesto al ingreso y un impuesto a los factores de producción. Como referencia de la vasta literatura se puede consultar Musgrave (1959), Mclure (1975), Break (1974) y Summers y Kotlikoff (1987).

Consideremos una función de producción (Q), con las conocidas propiedades de concavidad, en la que se utilizan como factores trabajo (L) y tierra $(T)$, esta última con una oferta fija:

$$
Q=F(L, T)
$$


Los cafetaleros maximizan su utilidad dependiendo de las ganancias que obtienen. Asumimos que la función de producción tiene rendimientos decrecientes a escala, de forma que los cafetaleros obtienen ganancias puras. Las ganancias en los diferentes sistemas de impuesto son:

$$
\begin{aligned}
& \pi_{\mathrm{x}}=\mathrm{P}\left(1-\tau_{\mathrm{x}}\right) \mathrm{Q}-\mathrm{WL}-\mathrm{MT} \text { Impuesto a las exportaciones } \\
& \pi_{p}=\left(1-\tau_{p}\right)(P Q-W L-M T) \text { Impuesto a las ganancias' } \\
& \pi_{1}=P Q-W L-\left(M+\tau_{1}\right) T \text { Impuesto a la tierra } \\
& \pi \text { : Ganancias } \\
& \text { P : Precio del café } \\
& \text { Q : Producción del café } \\
& \text { L : Trabajo } \\
& T \text { : Tierra } \\
& w \text { : Salario } \\
& \text { m : Renta } \\
& \tau \text { : Impuesto }
\end{aligned}
$$

En este modelo existen dos grupos: cafetaleros y trabajadores. Los impuestos recolectados por el gobierno no son compensados es decir no son redistribuidos. Los cafetaleros tiene aversión al riesgo. En la utilidad de ambos gnupos se asume que la primera derivada es mayor que cero y la segunda derivada es negativa con respecto al ingreso que obtienen.

La función de bienestar social se define como

$$
S W=a U_{G}+(1-a) U_{w}
$$

$U_{0}:$ Utilidad cafetaleros

$U_{w}:$ Utilidad trabajadores

a : Pesos asignados a la utilidad

En todo esta sección, se asume que los impuestos no son compensados, y el gobierno es neutral al riesgo. Las recaudaciones del goblemo, que dependen del tipo de impuesto, son:

$$
\begin{aligned}
& R_{x}=\gamma_{x} P Q, \text { con impuesto a las exportaciones } \\
& R_{p}=\gamma_{p}(P Q-W L-M T) \text {, con impuesto a las ganancias }
\end{aligned}
$$$$
R_{t}=\gamma_{t} T \text {, con impuesto a la tierra }
$$ 
Proposición 1: Asumiendo un modelo estático con certidumbre en los precios, tanto el impuesto a las ganancias como el impuesto a la tierra, son dominantes con relación al impuesto a la exportación, siendo los dos impuestos neutrales (lump-sum taxes) de cara a sus efectos distributivos y a sus efectos sobre la eficiencia. ${ }^{2}$ Comparado con los otros dos impuestos, el impuesto a las exportaciones es distorsionante y disminuye la función de bienestar social. Asl, los impuestos a la tierra $y$ al ingreso son alternativas superiores comparados con el impuesto a la exportación.

\section{EL IMPACTO DE LA INCERTIDUMBRE EN LOS PRECIOS DEL CAFE EN LA DEMANDA DE FACTORES}

Con incertidumbre con relación a los precios, la capacidad de toma de riesgos del gobierno y los cafetaleros modífica los resultados. En esta sección analizaremos el efecto de la incertidumbre en los precios sobre la demanda de factores.

Los cafetaleros maximizan una función de utilidad esperada, la cual depende de las ganancias. El precio es una variable aleatoria no degenerada con una media $\mu \Rightarrow E(P)=\mu$; es decir, la esperanza del precio es igual a $\mu$. En esta sección, seguimos un análisis similar a Sandmo (1971); la única ampliación que realizamos es el uso de los impuestos.

Proposición 2: La demanda de trabajo para el caso de incertidumbre en los precios es menor que en la situación de certidumbre.

Prueba:

Resolviendo el problema de maximización para el impuesto a la exportación, tenemos

$$
\begin{aligned}
& E\left[U^{\prime}\left(\pi_{x}\right)\left[P\left(1-\gamma_{x}\right) F_{L}-W\right]\right\}=0 \\
& E\left(U^{\prime}\left(\pi_{x}\right)\left[P\left(1-\gamma_{x}\right) F_{L}\right]\right]=E\left(U^{\prime}\left(\pi_{x}\right) W\right] \\
& U^{\prime}=\text { Diferencial de la función de utilidad } \\
& F_{L}=\text { Diferencial de la producción con respecto al trabajo. }
\end{aligned}
$$
obtiene

Restando $E\left(U^{\prime}\left(\pi_{x}\right)\left(1-\gamma_{x}\right) \mu F_{L}\right.$ ) en ambos lados de la igualdad, se

$$
E\left\{U^{\prime}\left(\pi_{x}\right)\left[(P-\mu)\left(1-\gamma_{x}\right) F_{L}\right]\right\}=E\left\{U^{\prime}\left(\pi_{x}\right)\left[w-\mu\left(1-\gamma_{x}\right) F_{L}\right]\right\}
$$


Sabemos que las ganancias del impuesto a la exportación vienen dadas por

$$
\pi_{x}=p\left(1-\gamma_{x}\right) Q-W L-M T
$$

Tomando la esperanza a esta expresión, obtenemos

$$
E\left(\pi_{x}\right)=\mu\left(1-\gamma_{x}\right) Q \cdot W L-M T
$$

Manipulando las últimas dos ecuaciones, se obtiene

$$
\pi_{x}=E\left(\pi_{x}\right)+(p-\mu)\left(1-\gamma_{x}\right) 0
$$

Debido a la concavidad de la función de utilidad, si $P>\mu$

$$
U^{\prime}\left[E\left(\pi_{\mathrm{z}}\right)\right]\left[(P-\mu)\left(1-\gamma_{\mathrm{L}}\right) F_{L}\right]>U^{\prime}\left(\pi_{\mathrm{x}}\right)\left[(P-\mu)\left(1-\gamma_{\mathrm{x}}\right) F_{\mathrm{L}}\right]
$$

Esta desigualdad también se mantiene en el caso en que $P<\mu$, porque la multiplicación por un número negativo en ambos lados revierte la desigualdad.

Al tomar la esperanza de la expresión anterior, su parte izquierda se hace cero, de modo que

$$
0>E\left\{U^{\prime}\left(\pi_{x}\right)\left[(P-\mu)\left(1-\gamma_{x}\right) F_{L}\right]\right\}
$$

Entonces, también se cumple que

$$
0>E\left\{U^{\prime}\left(\pi_{x}\right)\right\}\left[W-\mu\left(1-y_{x}\right) F_{L}\right]
$$

Por la concavidad de la función de utilidad, $U^{\prime}(\pi x)$ es siempre positiva; entonces, lo siguiente es cierto

$$
W \leq \mu\left(1-p_{x}\right) F_{L}
$$

Dado que hemos considerado salarios fijos, la demanda de trabajo - 0 reduce por la incertidumbre. Esto es originado por el hecho que la ganancia marginal esperada del factor excede su costo marginal. La producción y el trabajo son menores en la situación de incertidumbre. Soluciones similares resultan para el impuesto a la tierra y a las ganancias: la ganancia marginal del factor excede su costo marginal. Para el impuesto a las ganancias y a la tierra se puede probar que

$$
W \leq \mu F_{L}
$$




\section{EL IMPACTO DE LA INCERTIDUMBRE SOBRE LAS ALTERNA- TIVAS DE LA BASE DEL IMPUESTO AL CAFE}

Quedó probado anteriormente la reducción de la demanda de trabajo por la incertidumbre en los precios. En esta sección enfocamos el problema del impuesto óptimo. Los cafetaleros y el gobiemo toman decisiones considerando el hecho de que el precio no es conocido. Un supuesto importante es la aversión al riesgo por parte de los cafetaleros. Aun más, asumimos que su comportamiento es caracterizado por una aversión decreciente al riesgo absoluto. Las ventajas de ganancias más seguras cambian las opciones de la base del impuesto. No obstante, la utilidad es creciente dependiendo de las ganancias; la utilidad marginal es estrictamente decreciente a medida que las ganancias se incrementan. Las ventajas marginales en la utilidad por cambios en los ingresos son mayores cuando las ganancias son bajas.

Todas las decisiones de los agentes se toman antes de que se conozca el precio real. Asumamos que los precios siguen una distribución uniforme en el rango (Pmin, Pmax) y $E(P)=\mu$. El uso de esta distribución se justifica por dos razones: primero, las series de tiempo del precio del café muestran que éste ha venido fluctuando en un rango en el que no hay tendencia hacia un precio promedio; segundo, el uso de una distribución uniforme facilita el tratamiento analítico. El gobierno puede utilizar cualquiera de los impuestos. El bienestar social esperado es representado como un continuo de posibilidades ligadas a la probabilidad respectiva; las probabilidades son las mismas dado el supuesto de la distribución uniforme. ${ }^{3}$

$$
E(S W)=c \int_{P_{\min }}^{P_{\max }} U_{G}(P)+U_{W} d P
$$

1. El uso de un solo tlpo de Impuesto.

El método para evaluar la ganancia esperada, contiene los siguientes pasos. Primero, el gobierno selecciona una meta de ingresos fiscales. Segundo, las tasas de impuesto con respecto a esta meta son determinadas. Tercero, las tasas de impuesto son substituidas en la expresión correspondiente. Cuarto, el bienestar social es evaluado para comparar los beneficios de las diferentes políticas de impuestos.

Los impuestos óptimos bajos los distintos regimenes de impuesto son: 


$$
\begin{aligned}
& R^{\circ}=E(R) \\
& R^{\circ}=\gamma_{x} \mu Q^{4}
\end{aligned}
$$

El gobierno asume que el precio es la esperanza. Cuando la tasa del impuesto ha sido seleccionada, el nivel de producción es independiente del gobierno. Para obtener el impuesto óptimo $\left(\gamma_{x}{ }^{\circ}\right)$ una ecuación de alto orden en el impuesto tiene que ser resuelta, porque $Q$ es también función de $\gamma_{x}$.

La ganancia esperada a ser evaluada en la función de bienestar social es:

Impuesto a las exportaclones

$$
\pi_{x}=P\left(1-\gamma_{x}\right) Q-W L-M T
$$

Después de substituir la tasa de impuestos, obtenemos

$$
\begin{aligned}
& \pi_{x}=\left(1-R^{1} \mu Q_{x}\right) P Q_{x}-W L_{x}-M T \\
& \text { Impuesto a las Gananclas } \\
& R^{*}=\gamma_{p}(\mu \mathrm{Q}-W L-M T) \\
& O_{P}=\left(1-\gamma_{p}\right)(P Q-W L-M T) \\
& O_{P}=\left(1-R^{\prime} /(\mu Q-W L-M T)\right)(P Q-W L-M T) \\
& \text { Impuesto a la Tlerra } \\
& \begin{array}{l}
\mathrm{R}^{*}=\gamma_{t} \mathrm{~T} \\
\pi_{\mathrm{t}}=\mathrm{PQ}-\mathrm{WI}-\left(\mathrm{M}+\gamma_{t}\right) \mathrm{T} \\
\pi_{t}=\mathrm{PQ}-\mathrm{WL}-(\mathrm{M}+\mathrm{R} / T) \mathrm{T}
\end{array}
\end{aligned}
$$

Los precios representan un continuo. Tanto el gobierno como los cafetaleros trabajan con la esperanza; es decir, tienen que tomar declsiones antes de que el precio sea conocido. Ahora supongamos los dos casos extremos: que el precio sea muy bajo y que sea muy alto. En la situación de precios bajos, los cafetaleros obtienen más ganancias con el impuesto a las ganancias; en ese caso, el impuesto a 
la tierra es el más negativo para la utilidad de los cafetaleros. Si el precio es alto, los cafetaleros preferirán ser gravados con el precio a la tierra, siendo el menos deseable el impuesto a las ganancias. Para comprobar esto, simplemente comparemos la pendiente de la función de ganancias. Nótese la dependencia de los precios

$$
\begin{aligned}
& \left(1-R^{\circ} /(\mu Q-W L-M T)\right) Q<\left(1-R^{*} / \mu Q_{x}\right) Q_{x}<Q \\
& \text { Ganancias Exportación Tierra }
\end{aligned}
$$

Proposiclón 3: Las ganancias esperadas son maximizadas con el impuesto a las ganancias y a la tierra. El impuesto a las exportación genera menos ganancias esperadas.

Los impuestos a las ganancias reducen la varianza de los ingresos percibidos en la producción; para evaluar las ganancias, no tomamos en consideración la aversión al riesgo, de manera que los impuestos a la tierra y a las ganancias son equivalentes.

\section{Prueba:}

$$
\begin{aligned}
& \text { Evaluando las expresiones de ganancias para } P_{\max }=\mu+h \\
& \text { y } P_{\text {min }}=\mu-h \\
& \int_{\mu-h}^{\mu+h}\left(1-R^{\circ} / \mu Q_{x}\right) P Q_{x}-W L_{x}-M T d P \\
& 2 h\left(\mu Q_{x}-W L_{x}-M T\right)-2 h R^{\circ} \quad \text { Exportaciones } \\
& \int_{\mu-h}^{\mu+h}\left(1-R^{\circ} / \mu Q-W L-M T\right)(P Q-W L-M T) d P \\
& 2 h(\mu Q-W L-M T)-2 h R^{\circ} \quad \text { Ganancias } \\
& \int_{\mu-h}^{\mu+h} P Q-W L-\left(M+R^{\circ} / T\right) T d P \\
& 2 h(\mu Q-W L-M T)-2 h R^{\circ}
\end{aligned}
$$

Notemos que los impuestos a la tierra y a las ganancias obtienen resultados iguales. El impuesto a las exportaciones disminuye las ganancias esperadas porque $L_{x}<L$. $L$ es el nivel óptimo de trabajo: 
cualquier reducción disminuye las ganancias.

Q.E.D.

Los cafetaleros tienen mayor aversión al riesgo en el intervalo (Pmin, $\mu$ ), es decir, asignan más utilidad a no tener un bajo nivel de ganancias por precios bajos, que a tener un alto nivel de ganancias por precios favorables. La utilidad marginal de las ganancias se incrementan hasta $\mu$. Esto causa una diferencia al seleccionar la base óptima del impuesto. Para las mismas ganancias esperadas, un impuesto que permite a los productores tener mayores ganancias en el rango $\left(P_{m, n}, \mu\right)$ es preferido respecto a otro impuesto con las mismas ganancias esperadas.

Proposiclón 4: El impuesto a las ganancias es la forma más eficiente para obtener ingresos fiscales, dados los supuestos de aversión al riesgo de los cafetaleros y la incertidumbre en los precios.

\section{Prueba:}

El impuesto a la exportación es el más distorsionador; la reducción en la demanda de trabajo y la disminución en el ingreso de los trabajadores generan un bienestar social menor que el generado con el impuesto a la tierra o a las ganancias.

Tenemos que comparar los impuestos a las ganancias y a la tierra. Recordemos que ambos ocasionan el mismo ingreso para los trabajadores y la misma ganancia esperada.

Comparando el bienestar social esperado

$$
\begin{aligned}
& \int_{\mu-h}^{\mu+h} U\left(\pi_{P}\right)+U_{W} d P \quad \text { Ganancias } \\
& \int_{\mu-h}^{\mu+h} U\left(\pi_{p}\right)+U_{W} d P \quad \text { Tierra }
\end{aligned}
$$

Después de manipular los límites de integración y eliminar la utilidad de los trabajadores que es similar, probaremos que

$$
\int_{\mu-h}^{\mu+h} U\left(\pi_{P}\right)+U\left(\pi_{1}\right) d P>\int_{\mu-h}^{\mu+h} U\left(\pi_{1}\right)+U\left(\pi_{P}\right) d P
$$

La utilidad tiene menor pendiente en el segmento $(\mu, \mu+h)$; esto Implica que aumentos adicionales en las ganancias obtienen menor nlvel de utilidad. La pendiente de las ganancias es menor para el impuesto a las ganancias. 


$$
Q\left(1-R^{*} / \mu Q-W L-M T\right)<Q
$$

Los cafetaleros obtienen más ganancias en el intervalo $(\mu-h, \mu)$. Las diferencias en utilidad son más altas. Por consiguiente, la desigualdad se cumple.

\section{Q.E.D.}

\section{Disponibllidad de más de un Instrumento para gravar al cafó}

Para el caso de la disponibilidad de tres instrumentos, el problema es formulado de la manera tradicional: la maximización del bienestar social esperado sujeto a una meta de recolección fiscal. ${ }^{5}$

$$
\begin{aligned}
\operatorname{Max} E(S W)= & c \int_{P_{\min }}^{P_{\max }} U_{c}(y)+U_{w}(W L) d P \\
& \gamma_{x}, \gamma_{p}, \gamma_{t}
\end{aligned}
$$

Sujeto a

$$
R^{\cdot}=\gamma_{x} \mu Q+\gamma_{1} T+\gamma_{p}\left\{\left(1-\gamma_{x}\right) \mu Q-W L-\left(M+\gamma_{1}\right) T\right\}
$$

Las ganancias de los cafetaleros en este problema son

$$
\pi=\left(1-\gamma_{P}\right)\left\{\left(1-\gamma_{x}\right) P Q-W L-\left(M+\gamma_{1}\right) T\right\}
$$

La maximización restringida produce

$$
\begin{aligned}
& \int U_{Q}^{\prime}\left\{-\left(-1-\gamma_{p}\right) P Q-\left(1-\gamma_{p} \gamma_{p} \frac{\partial_{O}}{\partial_{L}} \frac{\partial_{L}}{\partial_{P_{X}}}-\frac{W \partial L}{\partial \gamma_{x}}\right\}\right. \\
& +U_{w}^{\prime}\left\{W \frac{\partial_{1}}{\partial \gamma_{x}}\right\} \\
& d P=h\left\{-\left(1-\gamma_{P}\right) \mu Q-\left(1-\gamma_{P}\right) \gamma_{x} \mu \frac{\partial_{O}}{\partial_{L}} \frac{\partial_{L}}{\partial_{\gamma_{x}}}\right\} \\
& \int U_{Q}{ }_{0}\left(1-\gamma_{x}\right) P Q-W L-\left(M+\gamma_{1}\right) T \\
& d P=h\left\{\left(1-\gamma_{n}\right) \mu Q-W L-\left(M+\gamma_{1}\right) T\right\} \\
& \int U_{Q}^{\prime}\left\{-T\left(1-\gamma_{p}\right)\right\}=h\left\{-T\left(1-\gamma_{p}\right)\right\}
\end{aligned}
$$


Después de resolver este sistema de ecuaciones no lineales, se obtienen las soluciones óptimas. El problema es que la ecuación para el impuesto a la exportación tiene expresiones de alto orden, y no hay garantia de solución única para el nivel óptimo de bienestar social. Este puede ser alcanzado combinando diversos impuestos. Después de encontrar las tasas óptimas de los impuestos, el bienestar social puede ser comparado con el obtenido con el uso de un solo tipo de impuesto. Comparando el costo relativo de incrementar una unidad de ganancia para cada uno de los impuestos, notamos que el impuesto a la exportación representa la forma más costosa de incrementar los ingresos fiscales del gobierno. Esto es por la distorsión que produce en la demanda de trabajo.

Proposiclón 5: Si el impuesto a las ganancias es utilizado, el valor óptimo de la tasa del impuesto a la exportación es cero.

\section{Prueba:}

De ser usado en combinación con el impuesto a las ganancias, el impuesto a la exportación reduce la ganancia de los cafetaleros en el intervalo en que la utilidad marginal se incrementa. El impuesto a las exportaciones puede representar una mejora Pareto en combinación con el impuesto a la tierra en los casos en que la varianza de los precios es grande.

Proposición 6: El uso del impuesto a las ganancias es superior en el sentido de Pareto de la combinación de impuestos a la ganancia y a la tierra.

\section{Prueba:}

Asumiendo que las tasas óptimas han sido obtenidas, expresamos el impuesto óptimo a las ganancias en función del impuesto a la tierra:

$$
\gamma_{p}=R^{\cdot}-\gamma_{1} T / \mu Q-W L-\left(M+\gamma_{1}\right) T
$$

La ganancia óptima es

$$
\pi=\left(1-R^{\circ}-\gamma_{1} T / \mu Q-W L-\left(M+\gamma_{1}\right) T\left(P Q-W L-\left(M+\gamma_{1}\right) T\right)\right.
$$

Dado que no existe distorsión en la demanda de trabajo y que los Ingresos del gobierno son iguales, la utilización de las combinación optima genera ganancias esperadas similares. Sin embargo, el beneficio social esperado disminuye, porque menos ganancias son obtenidas en 
el rango $(\mu-h, \mu)$, en donde la utilidad marginal de las ganancias se está incrementando. Para observar esto comparemos la pendiente de las ganancias.

$$
\left(1-R^{*} / \mu-W L-M T\right) Q<\left(1-R^{\circ}-\gamma T / \mu Q-W L-\left(M+\dot{\gamma}_{1}\right) T\right) Q
$$

Ahora consideraremos el supuesto de que el gobierno introduce subsidios. La presencia de subsidios es identificada mediante valores negativos en las tasas de impuestos.

Proposiclón 7: La solución óptima para el problema de la combinación adecuada incluye una combinación del impuesto a las ganancias y un subsidio a la tierra.

Prueba:

El impuesto a la exportación es eliminado por la distorsión que produce en la demanda de trabajo. Descentralizaremos la función de ganancias para demostrar la veracidad de la proposición.

$$
\left(1-\gamma_{P}\right)(P Q-W L)-\left(1-\gamma_{P}\right)\left(M+\gamma_{1}\right) T
$$

Notemos que cuando $p_{1}$ se hace negativa la función de ganancias es maximizada; y el compartir el riesgo garantiza un beneficio social mayor, dado que los cafetaleros están más protegidos de la eventualidad de precios desfavorables.

\section{UNA APLICACION CON UNA FUNCION DE UTILIDAD ISOELAS- TICA DE LOS CAFETALEROS}

En nuestro trabajo empirico asumiremos una función de utilidad de los cafetaleros con las siguientes caracteristicas

$$
U_{G}=\frac{(b+\pi)^{1 \alpha}}{1-\alpha}
$$

Donde b representa otras fuentes de ingresos de los cafetaleros. Esto asegura que el ingreso total de los cafetaleros siempre es positivo. El parámetro $\alpha$ captura la curvatura de la función de utilidad, asegurando asi el supuesto de absoluta aversión al riesgo de los cafetaleros.

La utilidad de los trabajadores tue representada por una función de 
elasticidad constante. La función de producción se asume que es Cobb-Douglas. A la elasticidad del trabajo se le asignó un valor de 0.5 . Esto no afecta nuestros resultados, dado que para cualquier valor el impuesto a las exportaciones siempre causa distorsión en la demanda de trabajo.

Después de encontrar las tasas de impuestos, se evalúa el beneficio social. Dimos los siguientes valores a nuestro modelo: $M=1, W=1$, $T=1$; el rango de los precios va de 1 a 20 , siendo 10 el valor esperado, dado nuestro supuesto de distribución uniforme.

Los resultados para diferentes recolecciones del gobierno y valores de 6 se muestran en cuadros 1, 2 y 3 . Los hallazgos empiricos sustentan nuestras proposiciones de la superioridad del impuesto a las ganancias sobre otros sistemas de impuestos.

CUADRO 1: BENEFICIO SOCIAL EN CADA TIPO DE IMPUESTOS

\begin{tabular}{|c|c|c|c|}
\hline \multicolumn{5}{|c|}{ Recolecclones del Goblemo $=10$} \\
\hline 0 & Exportaclones & Gananclas & Tlerra \\
\hline 1.20 & 23.89 & 50.41 & 48.08 \\
1.25 & 38.98 & 65.40 & 63.35 \\
1.50 & 62.39 & 89.20 & 87.96 \\
1.75 & 67.21 & 93.47 & 92.72 \\
2.00 & 68.31 & 94.54 & 94.08 \\
3.00 & 68.77 & 94.99 & 94.90 \\
5.00 & 68.78 & 95.00 & 94.98 \\
10.0 & 68.78 & 95.00 & 94.99 \\
\hline
\end{tabular}


CUADRO 2: BENEFICIO SOCIAL EN CADA TIPO DE IMPUESTOS

\begin{tabular}{|c|c|c|c|}
\hline \multicolumn{6}{|c|}{ Recolecciones del Goblemo = 5 } \\
\hline 6 & Exportaclones & Gananclas & Tlerra \\
\hline 1.20 & 40.08 & 50.97 & 50.08 \\
1.25 & 55.02 & 65.90 & 65.10 \\
1.50 & 78.49 & 89.30 & 88.91 \\
1.75 & 82.67 & 93.50 & 93.28 \\
2.00 & 83.71 & 94.50 & 94.43 \\
3.00 & 84.16 & 94.99 & 94.98 \\
5.00 & 84.17 & 95.00 & 94.99 \\
10.0 & 84.17 & 95.00 & 95.00 \\
\hline
\end{tabular}

CUADRO 3: BENEFICIO SOCIAL EN CADA TIPO DE IMPUESTOS

\begin{tabular}{|c|c|c|c|}
\hline \multicolumn{5}{|c|}{ Recolecclones del Goblerno = 5 } \\
\hline o & Exportaclones & Gananclas & Tlerra \\
\hline 1.20 & 49.40 & 51.30 & 51.14 \\
1.25 & 64.25 & 66.15 & 66.01 \\
1.50 & 87.50 & 89.40 & 89.39 \\
1.75 & 91.61 & 93.52 & 93.48 \\
2.00 & 92.64 & 94.54 & 94.52 \\
3.00 & 93.09 & 94.99 & 94.99 \\
5.00 & 93.10 & 95.00 & 95.00 \\
10.0 & 93.10 & 95.00 & 95.00 \\
\hline
\end{tabular}

\section{Conclusión}

Este ha sido un ejercicio teórico que ha sido validado con una simulación. Consideramos que si los problemas administrativos de la implementación de los impuestos mencionados pudieran ser minimizados, y que además de factibilidad representasen costos administrativos similares, podriamos sostener, con el fundamento demostrado a lo largo del articulo, que la manera más eficiente de gravar al café es a través de un impuesto a las ganancias de los cafetaleros. Esto se tra- 
duce a incorporar las ganancias de los cafetaleros al impuesto sobre la renta.

Es decir que asumiendo una administración adecuada nos atreveriamos a proponer que la forma más eficiente de gravar el café: es substituir el tradicional impuesto a la exportación por un impuesto sobre la renta. Se traduciría a declarar las utilidades provenientes del café como renta a personas naturales o jurídicas. Para que esto fuese factible se necesitarla que los costos de administración y la evasión se mantuvieran dentro de un límite. Implicaría también el diseno de tasas marginales acorde con la meta de recaudación programada, en una primera etapa tiene que buscarse una recaudación similar. Esto llevaria a modificar tasas y tramos de ingresos del impuesto sobre la renta, medida que puede distorsionar la estructura existente del impuesto sobre la renta.

Mientras no se mejore la administración tributaria la forma más conveniente de recaudación es el tradicional impuesto a la exportación. Una recomendación de política fiscal en la dirección de transferir el impuesto a la exportación de café a impuesto sobre la renta asume una profunda transformación en la administración y diseno del impuesto sobre la renta.

\section{NOTAS}

1. Notemos que el impuesto a las ganancias por ser proporcional no afecta las decisiones de contratar trabajo. Lo mismo que el impuesto a la tierra por recaer solo sobre el factor tierra.

2. Lumi-sum takes o impuestos de transferencia compensada son aquellos que no causan ninguna distorsión.

3. Los pesos de las utildiades se asume que son 1 para simplificar la formulación.

4. El gobierno y los cafetaleros toman decisiones asumiendo que el precio esperado es $\mu$.

5. El impuesto óptimo aqul es más cercano al problema de impuesto lineal óptimo que a la solución de Ramsey. Aunque tiene diferentes componentes que el problema tradicional de la solución óptima del problema del impuesto al ingreso.

\section{BIBLIOGRAFIA}

Break. G.F. (1974), "The Incidence and Economic Effects of Taxation", en A. Blinder et. al. eds. The Economics of Public Finance. Brookings Institution.

McLure, C.E. (1975), "General Equilibrium Incidence Analysis: The Harberger Model after Ten Years". Journal of Public Economics 4, 125-161. 
Musgrave, R.A. (1959), The Theory of Public Finance. Mc Graw-Hill.

Sandmo, A. (1971), "On the Theory of the Competitive Firm under Price Uncertainty". American Economic Review 61, 65-75.

L. Summers y L. Kotlikoff (1987), "Tax Incidence". Handbook of Public Economics Vol. II, 1043-1092. 